\title{
Forearm Loss Caused by Automated Non-Invasive Blood Pressure Cuff Malfunction: A Hearsay Report
}

\author{
Steven M. Shulman 1*, Yunseok Namn², Stanislav Lando ${ }^{3}$, Patrick Discepola4 \\ ${ }^{1}$ Department of Anesthesiology, Rutgers-New Jersey Medical School, Newark, USA \\ ${ }^{2}$ Department of Anesthesiology, Department of Anesthesiology, Yale University, New Haven, USA \\ ${ }^{3}$ Department of Anesthesiology, Montefiore Medical Center, New York, USA \\ ${ }^{4}$ Department of Anesthesiology, Wake Forest Baptist Medical Center, Winston-Salem, USA \\ Email: "steve@steveshulman.com
}

Received 6 October 2015; accepted 6 November 2015; published 9 November 2015

Copyright (C) 2015 by authors and Scientific Research Publishing Inc.

This work is licensed under the Creative Commons Attribution International License (CC BY).

http://creativecommons.org/licenses/by/4.0/

(c) (i) Open Access

\begin{abstract}
Failure of an automated blood pressure cuff to deflate when a patient is under general anesthesia can lead to catastrophic consequences if unnoticed for more than three hours [1]. We present this as a hearsay case in which an automated blood pressure cuff of the Spacelabs Ultraview Clinical Workstation monitor (model No. 90385) applied pressure for about five hours resulting in limb thrombosis. In order to analyze this catastrophe, simulation scenarios were tested to elucidate the possible errors and malfunctions that may have led to this injury. We present the analysis of the advantages and validity of the hearsay case report. We also include our proposed criteria that should be required when a hearsay case is considered for publication.
\end{abstract}

\section{Keywords}

Monitor, Malfunction, Hearsay, Compartment Syndrome, Non-Invasive Blood Pressure

\section{Introduction}

The malfunction of an automated non-invasive blood pressure monitor (NIBP) which caused the cuff to remain inflated for greater than five hours caused the loss of an arm below the level of the cuff. While compartment syndrome is a recognized complication of NIBP monitoring [2], the initial question is how this malfunction could go unnoticed by the experienced anesthesiologist who was caring for the patient. The NIBP monitor is

\footnotetext{
${ }^{*}$ Corresponding author.
} 
usually used to measure blood pressure, but also has a venous tourniquet setting that maintains a pressure of 60 mmHg for 2 - 5 minutes in order to facilitate intravenous (i.v.) catheterization. The "venous stasis" (VS) setting in the NIBP menu on the Spacelabs monitor model 90385 inflates the cuff for two minutes. The malfunction of a venous compression pressure cuff may lead to catastrophic results that may be not apparent to the anesthesiology caretakers.

We have heard through a clear second hand report that the events described herein actually occurred in 2011 although we do not know the exact cause of the failure to deflate. We also did not have access to the actual records. Of note, Spacelabs Healthcare terminated guaranteed service for this monitor in 2010 since it had not been manufactured for seven years [3]. We believe there are important safety issues which still place patients at risk four years after the incident; hence we present it as hearsay case to alert anesthesiologists of the potential adverse situation that may occur.

\section{Materials and Methods}

The monitor involved in the actual case was the Spacelabs Monitor model 90385. In order to determine how these events may have occurred, with IRB approval, we have tested various conditions on ourselves with the Phillips Intellivue MP70 monitor to determine what clinical factors placed the patient at risk or obscured the threat to the clinician. This model was used was chosen for the simulation as it was available to us. We emphasize that the Phillips product was not involved in the actual case. The case was discussed with both Philips and Spacelabs technical support. The following scenario was developed so that a blood pressure cuff remaining inflated throughout a procedure under general anesthesia would go unnoticed by the clinician.

Internet searches were performed with two goals. First, the question of whether limb loss has been previously reported due to NIBP malfunction was addressed. Secondly, whether a hearsay report had been used to publish a medical complication prior to this report was also explored. The internet search engines used were Google, Pub Med, Bing, Yahoo search, AOL Search, Web Crawler, and Web MD with the keywords: Monitor, malfunction, hearsay, Compartment syndrome, and Non-invasive blood pressure. The depth of the search was five internet pages.

\section{Simulation Description}

A patient was brought to the operating room for an operation under the care of an experienced anesthesiologist with major fluid shifts and the potential for hemodynamic instability. General anesthesia was induced without complication. The blood pressure cuff which had been applied correctly was then inflated on venipuncture mode to $60 \mathrm{mmHg}$ in order to facilitate the placement of an additional intravenous catheter. Once the intravenous line was secured, as the fluid bag was about $84 \mathrm{~cm}$ higher than the NIBP cuff, the flow was brisk and the cuff never deflated. Subsequently, an arterial catheter was placed in the contralateral arm which also had been used for the pulse oximeter probe. Lastly an upper body warming blanket was secured, covering both arms. The blood pressure was recorded from the arterial catheter pressure reading. The MAP of "(60)" displayed under the NIBP reading was disregarded as an old NIBP reading.

After five hours, upon removing the surgical drapes, the arm with the NIBP cuff was inspected and noted to be dark, cold and pulseless, distal to the inflated blood pressure cuff. An emergent angiogram revealed extensive thrombosis of the limb's arteries and veins. The vascular interventions to the limb proved to be futile; the arm was unable to be reperfused, dry gangrene set in and the limb was eventually amputated.

\section{Discussion}

In the literature, compartment syndrome due to NIBP malfunction has been attributed to excessive cycling due to a tremor [4] or malposition of the NIBP cuff over the antecubital fossa [5]. There is also a report of rhabdomyolysis following frequent NIBP cycling during a six hour case [6]. The simulation scenario assumed that neither tremor nor malposition of the NIBP cuff. The simulation scenario demonstrated that failure of the NIBP to deflate can go unobserved when five specific conditions that occurred under routine clinical care. First, the i.v. fluid flowed when the crystalloid bag was $84 \mathrm{~cm}$ higher than the limb. Secondly, the arterial line and the pulse oximetry probe were both placed on the contralateral limb so that the opposite hand is monitored by the pulse oximeter for low hand blood flow due to the arterial catheter which is typical for clinical practice. Third, the 
blood pressure cuff was never triggered after the arterial catheter was placed so that the fail to inflate was not noticed and investigated. There was no alarm to alert the practitioner to presence of the inflated cuff. Fourth, the upper body warming blanket covered the upper limbs. In our scenario, the use of the upper body warmer concealed the skin changes associated with the ischemia. Finally, the clinician did not routinely inspect the limbs.

As always, when monitor failure is suspected, the clinician's first priority is to ensure the patient's well-being. Usually, monitor failure is only a distraction. Here the monitor failure posed a direct threat to the patient. Obviously, patients who have their arms tucked in at their sides would be difficult to inspect. Likewise, chest roll migration in a patient in the prone position has been reported by Lee et al. [7]. For clinicians, we recommend routine inspection of hands when possible especially for patients in prone position.

The three causes for the blood pressure cuff to not deflate are either that the blood pressure module malfunctioned, debris in the system obstructed deflation or the tubing was kinked. In the simulation scenario, the clinician relied on the arterial catheter pressure readings so the tubing, BP cuff and the module were never checked for continuous inflation. The Spacelab Manual explicitly describes that in event the cuff cannot be deflated, "Remove the cuff from the limb immediately and have the module serviced by biomed or a qualified field service engineer" [8]. Even cycling the BP cuff every 60 minutes may not have deflated the cuff if it was due to module malfunction, but this may have possibly alert the clinician to the failure of the cuff. Alternatively, it may potentially clear debris obstructing the outflow of the gas keeping the cuff inflated.

Likewise, the Philips manual also mentions the possibility that "kinked or otherwise restricted tubing can lead to a continuous cuff pressure, causing blood flow interference and potentially resulting in injury to the patient." In that manual they recommend to "Inspect the application site regularly to ensure skin quality and inspect the extremity of the cuffed limb for normal color, warmth and sensitivity. If the skin quality changes, or if the extremity circulation is being affected, move the cuff to another site or stop the blood pressure measurements immediately" [9]. The Phillips monitor also displays the venipuncture icon "VP" above the MAP reading so that it cannot be mistaken for the previous BP. However we found this symbol small and easily ignored.

Very low frequency events such as this should be reported in order to increase clinical vigilance to avoid their repetition. Fortunately, malfunction of a NIBP monitor is rare. The Thai Anesthesia Incidents Study estimated the incidence of equipment malfunction/failure at 3.4 per 10,000 [10]. After using major search, we were unable to find a report in the medical literature of failure of a blood pressure cuff to deflate that resulted in limb loss. Of note, we did find one request on the internet looking for an expert witness describing a legal case dealing with postoperative blood pressure malfunction in which the cuff was inflated for three days resulting in a crush injury [11]. We assume that the inflated cuff would cause excruciating tourniquet or ischemic pain in a conscious patient so it is likely that this patient was either sedated or comatose. Furthermore, we could find no prior reference of a hearsay case in medical literature. We believe this is the first hearsay clinical report published.

The traditional medical case report requires a motivated author unafraid of the legal consequences of publishing the case involving a disastrous clinical event. A clinician after a clinical catastrophe may be liable to a potential civil lawsuit and so is unlikely to publish the case at least until the statute of limitations has expired. This delay may result in further adverse events. A catastrophic complication is not only traumatic to the patient and family, but to the clinician involved, a consequence of medical error that is all too often not addressed. This may make it emotionally difficult for the involved clinicians to take the time and effort to publish such an error. Wu and Steckelberg describe these physicians involved in medical error as "second victims" who do not have access to professional reaffirmation and personal reassurance that is offered to "first victims" such as the patient and family members [12]. Furthermore, those physicians may fear scorn or derision from their colleagues. Hospitals are understaffed and resource underutilized when it comes to delivering emotional aid when serious incidents occur. Even if an adverse event of a very low frequency where to be reviewed by an anesthesiology quality assurance committee, the internal review of such events is not published or discussed publicly thus preventing dissemination of knowledge. We believe the hearsay report provides a means for an outlet for the clinician as hospitals continue work towards a policy that allows for an open and safe disclosure of adverse events.

Another reason a catastrophic case of instrument malfunction may be unlikely to be published as a case report is that it raises concerns about the maintenance of the equipment by its owners. The product involved is the Ultraview Clinical Workstation (UCW product \#90385) which was last manufactured in January 2003 [3], so it was at least eight years old. The service contract guarantees support coverage for a period of 7 years, until January 2010 from which at that point, coverage would be transitioned to "best effort support." As this incident is known to have occurred in 2011, the manufacturer had no longer guaranteed support. Best efforts is a contrac- 
tual term used to obligate the parties to make their best attempt to accomplish a goal, typically used when there is uncertainty about the ability to meet the goal. Courts have not required that a party under a duty to use best efforts to accomplish a given goal make every available effort to do so, regardless of the harm to it.

We propose that publishing hearsay case report is a way for clinical knowledge that has been communicated verbally to inform the wider audience of the written literature when hurdles exist for its immediate publication. Underreporting of adverse events occurs with the incident reporting system [13]. A hearsay report is not "evidence based medicine" [14]. Nonetheless, medical knowledge is a product of both a proud oral tradition and extensive written history. We have researched the medical literature and have found no discussion of hearsay publication that occurs in medicine. Hearsay accounts are part of this oral tradition permitting the opportunity for us to hear of other's errors, much of which occurs through word of mouth. Hearsay encompasses evidence that does not arise from one's own personal account of a critical event but rather through hearing what others say [15]. The strength in a hearsay report is that it allows us a "real time" view of a mishap in medicine. It captures the present day events through which the meanings and practice of medicine are constantly being created, molded, and changed "in situ" that can only be expressed through the publication of individual experiences and interview situated accounts [16]. The event described here happened in 2011, but we believe that the danger still exists and there is no report of a similar event that has yet been published. Despite not being evidence-based medicine, the authors believe hearsay is an invaluable means of communication if it means potentially saving a patient's life or in this case, a limb. We also strongly believe that it should only be used rarely when no other means of publication are likely. To our knowledge, this is the first reported case of hearsay in the anesthesiology literature that is clearly identified as such.

We believe this is the first case to be presented in the hearsay form. The authors recognize the potential problem that hearsay cases present to editors and journals in that they may become inundated with repetitious and facetious submissions of third or fourth hand, poorly described case studies. Hence we agree it should only be used sparingly and as a last resort.

For hearsay cases, the authors have developed eight criteria that must be first met before publication:

1) Authors must present a case based on factual events.

2) Authors must have a source connected to the parties involved of the actual case. The report must be second hand and not simply gossip of an unknown source.

3) Authors must be able to identify the location and approximate time of event.

4) The case reported must be unique and involve a previously undescribed issue. It should only be used to warn clinicians of a harmful situation. It should never be used to advocate a technique or a clinical management guideline without an accompanying expert consensus.

5) There must be clear and present danger to patients without the publication of the report.

6) Authors must present a case that is not likely to be soon published.

7) Authors must be able to identify key features of the event.

8) Authors must have no ulterior motives or mal-intent in the publication of the report.

An additional ninth criterion for the publication is proposed of what can be referred to as a "proxy case report". The case report may also be reported by other clinicians who receive all the records from the original source. In the event that consent can be attained from one of clinicians involved in the case, then the publication should be considered as a report by proxy and thus would be superior to being reported as a hearsay report.

We propose that the one advantage of a hearsay report over closed claims analysis is its rapidity. The strength of the laudable closed claims study analysis lies in the analysis by an impartial third party of the closed claims as well as the ability to "overcome" the hesitancy to publish cases that have poor outcomes. The closed claims analysis depends on the case entering the closed claims database. Years may pass before these critical events are examined. Some injured patients may not even file claims. A hearsay report may inform clinicians without delay of waiting for a case to be settled or statute of limitations to expire. Closed claims studies can take at least 2 years to 5 years between the actual occurrence of the critical event and the actual closure of the associated claim in order for it to be available for review [17]. Hearsay cases offer immediate reporting of errors and acts as an early warning system to prevent similar potential errors from occurring in the future. It is our hope that hearsay cases can be reported with increased legal liability to the potential defendants. The institution and clinicians involved in an actual case may be facing possible legal action so they may be reluctant to make public statements that may have ramifications in the courtroom and in legal actions. Other clinicians in the vicinity may also try to psychologically distance themselves from a catastrophe often characterized by such comments as “I wasn't on 
when it happened”, or “It wasn’t my case”. Lastly some may want to discourage publication of a poor outcome because they consider this will negatively impact their reputation by "advertising” their mistake.

Clinicians are inherently historians. We are taught from the beginning of our training to record clinical observations. This training emphasizes accuracy in details. Hearsay to the clinician is considered a poor substitute for a first person clinical report. While the hearsay case report is an inferior substitute to a first-hand case report, we intend it here to be an early warning for clinicians. We have defined the criteria for a hearsay case so it is not simply gossip transcribed. For the medical editor, this is a concern that the acceptance of hearsay reports will lead to a proliferation of poor, inaccurate case reports. We believe there is a role, albeit a rare one for the publication of a hearsay case.

\section{Conclusion}

In conclusion, five clinical events had to occur so that the inflated blood pressure cuff would go unnoticed by the experienced clinician. This is consistent with the Swiss cheese model of system accidents where multiple hazardous conditions are present when an adverse clinical event occurs [18]. Also, we believe that routine inspection of the hands, when possible, to check for evidence of poor circulation should be considered.

\section{References}

[1] Kam, P.C.A., Kavanaugh, R. and Young, F.F.Y. (2001) The Arterial Tourniquet: Pathophysiological Consequences and Anesthetic Implications. Anaesthesia, 56, 534-545. http://dx.doi.org/10.1046/j.1365-2044.2001.01982.X

[2] Schroeder, B., Barbeito, A., Bar-Yosef, S. and Mark, J.B. (2014) Cardiovascular Monitoring. Chap 45. Miller’s Anesthesia. In: Miller, R.D., Ed., 8th Edition, 1349.

[3] Spacelabs Healthcare Service Discontinuation Notification (2009) Support of Spacelabs Healthcare Patient Monitoring Products. 1-2.

[4] Celoria, G., Dawson, J.A. and Teres, D. (1987) Compartment Syndrome in a Patient Monitored with an Automated Blood Pressure Cuff. Journal of Clinical Monitoring, 3, 139-141. http://dx.doi.org/10.1007/BF00858363

[5] Sutin, K.M., Longaker, M.T., Wahlander, S., Kasabian, A.K. and Capan, L.M. (1996) Acute Biceps Compartment Syndrome Associated with the Use of a Noninvasive Blood Pressure Monitor. Anesthesia \& Analgesia, 83, 1345-1346.

[6] Srinivasan, C. and Kuppuswamy, B. (2012) Rhabdomyolysis Complicating Non-Invasive Blood Pressure Measurement. Indian Journal of Anaesthesia, 56, 428-430. http://dx.doi.org/10.4103/0019-5049.100847

[7] Lee, J.A., Jeon, Y.S., Jung, H.S., Kim, H.G. and Kim, Y.S. (2010) Acute Compartment Syndrome of the Forearm and Hand in a Patient of Spine Surgery-A Case Report. Korean Journal of Anesthesiology, 59, 53-55. http://dx.doi.org/10.4097/kjae.2010.59.1.53

[8] Spacelabs Healthcare, LLC. Spacelabs Ultraview SL ${ }^{\text {TM }}$ Operations Manual 070-1150-01, Rev. AB. Sect 16-16, 260.

[9] Phillips USA (2015) Phillips Instructions for Use Intellivue Patient Monitor MP20/30, MP40/50, MP60/70/80/90. Release K with Software Revision K.2x.xx Patient Monitoring, 223.

[10] Charuluxananan, S., Punjasawadwong, Y., Suraseranivongse, S., Srisawasdi, S., Kyokong, O., Chinachoti, T., Chanchayanon, T., Rungreungvanich, M., Thienthong, S., Sirinan, C. and Rodanant, O. (2005) The Thai Anesthesia Incidents Study (THAI Study) of Anesthetic Outcomes: II. Anesthetic Profiles and Adverse Events. Journal of the Medical Association of Thailand, 88, S14-S29.

[11] Talve, M. (2013) Blood Pressure Cuff Malfunction Causes Crush Syndrome. https://www.theexpertinstitute.com/case-studies/blood-pressure-cuff-malfunction-causes-crush-syndrome/

[12] Wu, A.W. and Steckelberg, R.C. (2012) Medical Error, Incident Investigation and the Second Victim: Doing Better but Feeling Worse? BMJ Quality \& Safety, 21, 267-270. http://dx.doi.org/10.1136/bmjqs-2011-000605

[13] Sari, A.B.A., Sheldon, T.A., Cracknell, A. and Turnbull, A. (2007) Sensitivity of Routine System for Reporting Patient Safety Incidents in an NHS Hospital: Retrospective Patient Case Note Review. BMJ, 334, 79. http://dx.doi.org/10.1136/bmj.39031.507153.AE

[14] Dipaola, R.S. and Gallo, M.A. (2008) Hearsay Medicine Is Not Evidence-Based Medicine. Clinical Cancer Research, 14, 337-338. http://dx.doi.org/10.1158/1078-0432.CCR-07-2237

[15] Coleman, A.H. (1965) The Hearsay Evidence Rule and the Physician. Journal of the National Medical Association, 57, 256-257.

[16] Watkins, S.C. and Swidler, A. (2009) Hearsay Ethnography: Conversational Journals as a Method for Studying Culture in Action. Poetics (Amst), 37, 162-184. http://dx.doi.org/10.1016/j.poetic.2009.03.002 
[17] Lee, L.A. and Domino, K.B. (2002) The Closed Claims Project. Has It Influenced Anesthetic Practice and Outcome? Anesthesiology Clinics North America, 20, 485-501. http://dx.doi.org/10.1016/S0889-8537(02)00006-8

[18] Reason, J. (2000) Human Error: Models and Management. BMJ, 320, 768-770.

http://dx.doi.org/10.1136/bmj.320.7237.768 